\title{
More Information on the Reproducibility of the Ambulatory Arterial Stiffness Index
}

\author{
Yan Li ${ }^{1}$, Tine W. Hansen ${ }^{2}$ and Jan A. Staessen 3,4
}

( n 2006, based on physiologic principles already published in $1914,{ }^{1}$ we defined the ambulatory arterial stiffness index (AASI) as 1 minus the regression slope of diastolic on systolic blood pressure (BP) in individual 24-h ambulatory $\mathrm{BP}$ recordings. ${ }^{2}$ AASI is an integrated measure, which is characteristic for an individual, and which reflects the combined effects of left ventricular ejection, active and passive components of arterial stiffness, and the reflection of the arterial pulse wave. The stiffer the arterial tree, the closer the regression slope is to 0 and AASI is to 1 .

In this issue of the journal, Stergiou and colleagues reported on the reproducibility of AASI at an interval of 2-4 weeks in 126 untreated hypertensive patients. ${ }^{3}$ Mean values of AASI were similar in first and repeat ambulatory recordings. The repeatability coefficient of AASI expressed as a percentage of near maximal variation was 50\%. Stergiou's findings ${ }^{3}$ are in keeping with those reported by us at an 8-day interval in 152 patients with systolic and diastolic hypertension (55\%) and at a 31-day interval in 145 patients with isolated systolic hypertension $(61 \%){ }^{4}$ Stergiou and colleagues estimated the repeatability coefficient of AASI from published statistics available in other reports. It was $58 \%$ in 38 healthy volunteers at a 2 -week interval, ${ }^{5}$ and $55 \%$ and $58 \%$ in 568 normotensive subjects and 211 hypertensive patients, respectively, at a 2 -year interval. ${ }^{6}$ By and large, the findings from all studies ${ }^{3-6}$ show that AASI has moderate repeatability, comparable with that of the morning surge in BP (52-75\%), ${ }^{7}$ but lower than the repeatability of the 24-h BP level (30\%). ${ }^{4}$ Higher values of the repeatability coefficient indicate lower reproducibility.

Stergiou and colleagues also reported the repeatability of AASI computed from daytime (69\%) and night time (74\%) recordings. We do not recommend to compute AASI for daytime and night time separately, because fewer BP readings over a narrower $\mathrm{BP}$ range result in erratic estimates of the

\footnotetext{
${ }^{1}$ Center for Epidemiologic Studies and Clinical Trials, Ruijin Hospital, Shanghai Jiaotong University School of Medicine, Shanghai, China; ${ }^{2}$ Research Center for Prevention and Health and Department of Clinical Physiology, Hvidovre University Hospital, Faculty of Health Sciences, Copenhagen, Denmark; ${ }^{3}$ The Studies Coordinating Centre, Division of Hypertension and Cardiovascular Rehabilitation, Department of Cardiovascular Diseases, University of Leuven, Leuven, Belgium; ${ }^{4}$ Department of Epidemiology, Maastricht University, Maastricht, The Netherlands. Correspondence: Jan A. Staessen (jan.staessen@med.kuleuven.beor ja.staessen@epid.unimaas.nl)

doi:10.1038/ajh.2009.239

(c) 2010 American Journal of Hypertension, Ltd.
}

regression slope of diastolic on systolic BP. A worse fit of the AASI regression line results in lower reproducibility, as also exemplified by Stergiou's results. ${ }^{3}$ In our Chinese population study $(n=348),{ }^{1}$ the correlation coefficients of the central and peripheral augmentation indexes with AASI were substantially weaker for the daytime AASI $(r=0.29$ and 0.09) and night time AASI ( $r=0.32$ and 0.09$)$ than for the 24-h AASI $(r=0.48$ and 0.50). Along similar lines, in 166 Chinese volunteers, ${ }^{1}$ the correlation of pulse wave velocity with daytime AASI $(r=0.18)$ and night time AASI $(r=0.22)$ was lower than that with 24 -h AASI $(r=0.51)$. Ben-Dov proposed symmetrical AASI ${ }^{8}$ as an alternative to our definition of AASI, ${ }^{1}$ because it would be less affected by the nocturnal dipping of BP. However, Stergiou's study ${ }^{3}$ highlighted that in terms of reproducibility symmetrical AASI does not offer any advantage over usual AASI. For reasons argued elsewhere, ${ }^{9}$ we suggest not to use symmetrical AASI, unless prospective studies would show that it is a better predictor of all and cause-specific fatal and nonfatal cardiovascular outcomes than AASI as initially defined. ${ }^{1}$

One limitation of Stergiou's study, as stated in the Discussion, ${ }^{3}$ was that he did not address the issue to what extent the fit of the AASI regression line, as expressed by the coefficient of determination $\left(r^{2}\right)$ affects the reproducibility of AASI. We demonstrated that a higher goodness of fit of the AASI regression line in individual subjects strengthens the association with the known ${ }^{1}$ determinants of AASI. ${ }^{4}$ Indeed, the $z$-score for the association of AASI with four determinants of AASI combined (age, height, mean arterial pressure, and heart rate) increased curvilinearly with $r^{2}$, with most of the increase occurring above the 20th percentile of $r^{2}(0.36)$. We found lower repeatability, when in each of two repeat recordings $r^{2}$ was $<0.36$ as opposed to $\geq 0.36$ (repeatability coefficients $68 \%$ vs. $44 \%$, respectively).

In line with other studies, ${ }^{4-6}$ Stergiou and colleagues ${ }^{3}$ demonstrated that AASI has only limited reproducibility. However, what clinically counts most, is that AASI improves the risk stratification based on ambulatory BP monitoring. To date, several cross-sectional studies and at least three prospective cohort studies have demonstrated association of AASI with either signs of target organ damage, or with incidence of cardiovascular mortality and morbidity. ${ }^{10}$ When adjusted for pulse pressure or pulse wave velocity, AASI remained predictive, in particularly of stroke. ${ }^{10}$ Thus the moderate reproducibility of AASI did not preclude its ability 
to be associated with target organ damage or to predict outcome. ${ }^{10}$ Although AASI was introduced only 3 years ago, ${ }^{1}$ a PubMed search using the term "ambulatory arterial stiffness index" at the time of writing of this Commentary yielded close to 70 hits. Moderate reproducibility is therefore unlikely to affect the interest in AASI for use in clinical practice and epidemiologic research.

\section{Disclosure: The authors declared no conflict of interest.}

1. MacWilliam JA, Melvin GS. Systolic and diastolic blood pressure estimation with special reference to the auditory method. BrMed J. 1914; 28:693-697.

2. Li Y, Wang JG, Dolan E, Gao PJ, Guo HF, Nawrot T, Stanton AV, Zhu DL, O'Brien E, Staessen JA. Ambulatory arterial stiffness index derived from 24-hour ambulatory blood pressure monitoring. Hypertension 2006; 47:359-364.

3. Stergiou GS, Kollias A, Rarra VC, Roussias LG. Ambulatory Arterial Stiffness Index: reproducibility of different definitions. Am J Hypertens 2010;23:129-134.

4. Dechering DG, van der Steen MS, Adiyaman A, Thijs L, Deinum J, Li Y, Dolan E, Akkermans RP, Richart T, Hansen TW, Kikuya M, Wang J, O'brien E, Thien T,
Staessen JA. Reproducibility of the ambulatory arterial stiffness index in hypertensive patients. J Hypertens 2008; 26:1993-2000.

5. Gosse P, Papaioanou G, Coulon P, Reuter S, Lemetayer P, Safar M. Can ambulatory blood-pressure monitoring provide reliable indices of arterial stiffness? Am J Hypertens 2007; 20:831-838.

6. Gosse P, Roche F, Dauphinot V, Maudoux D, PichotV, Barthelemy JC. Components of arterial stiffness in a population of 65-year-old subjects: PROOF study. J Hypertens 2008; 26:1138-1146.

7. Wizner B, Dechering DG, Thijs L, Atkins N, Fagard R, O'Brien E, de Leeuw PW, Parati G, Palatini P, Clement D, Grodzicki T, Kario K, Staessen JA. Short-term and long-term repeatability of the morning blood pressure in older patients with isolated systolic hypertension. J Hypertens 2008; 26:1328-1335.

8. Ben-Dov IZ, Gavish B, Kark JD, Mekler J, Bursztyn M. A modified ambulatory arterial stiffness index is independently associated with all-cause mortality. J Hum Hypertens 2008; 22:761-766.

9. Adiyaman A, Dechering DG, Thien T, Boggia J, Hanssen TW, LiY, Wang JG, O'Brien E, Staessen JA. Putting a spin on the ambulatory arterial stiffness index. J Hypertens 2008; 26:1266-7; author reply 1267.

10. Hansen TW, LiY, Staessen JA, Jeppesen J, Rasmussen S, Wang JG, Thijs L, Ibsen H, Safar ME, Torp-Pedersen C. Independent prognostic value of the ambulatory arterial stiffness index and aortic pulse wave velocity in a general population. J Hum Hypertens 2008; 22:214-216. 